Efficacy of Lurasidone in Antipsychotic-Naive vs. Antipsychotic-Exposed Adolescents with Schizophrenia: Post-Hoc Analysis of a Two-Year, Open-Label Study

Christoph Correll, $\mathrm{MD}^{1}$, Michael Tocco, $\mathrm{PhD}^{2}$, Andrei Pikalov, MD, $\mathrm{PhD}^{2}$, Jay Hsu, $\mathrm{PhD}^{2}$ and Robert Goldman, $\mathrm{PhD}^{2}$

\footnotetext{
${ }^{1}$ The Zucker Hillside Hospital, Department of Psychiatry, Northwell Health, Glen Oaks, NY, USA; Hofstra Northwell School of Medicine, Department of Psychiatry and Molecular Medicine, Hempstead, NY, USA; and Charité Universitat Medizin, Department of Child and Adolescent Psychiatry, Berlin, Germany, and ${ }^{2}$ Sunovion Pharmaceuticals Inc., Fort Lee, NJ and Marlborough, MA, USA
}

Presenting Author: Michael Tocco

\section{Abstract}

Background. Few studies have examined treatment response in adolescents with schizophrenia who are treatment-naive; and there is no placebo-controlled study that we are aware of in first episode treatment-naive patients with schizophrenia. The aim of this analysis was to evaluate the long-term efficacy of lurasidone in antipsychotic-naive adolescents with schizophrenia.

Method. Patients aged 13-17 years with schizophrenia, and a PANSS total score $\geq 70$ and $<120$, were randomized to 6 weeks of double-blind (DB) treatment with lurasidone (40 or $80 \mathrm{mg} /$ day) or placebo. Six-week completers were eligible to enroll in a 2 -year open-label extension phase receiving lurasidone flexibly dosed from $20-80 \mathrm{mg} /$ day. In a post-hoc analysis, efficacy was evaluated for 2 patient groups based on treatment status prior to entering the initial 6-week DB study (treatment naïve [TN] vs. treated previously [TP]). Treatment-naïve was defined as never having received antipsychotic treatment. Efficacy measures included the PANSS total score and the Clinical Global Impression, Severity (CGI-S) score. Level of functioning was assessed using the Children's Global Assessment Scale (CGAS), with a score of 70 representing normative levels of functioning.

Results. A total of $50 \mathrm{TN}$ and $221 \mathrm{TP}$ patients completed the 6-week DB study and entered the extension study; and 30 (60.0\%) TN and 126 (57.0\%) TP patients completed 104 weeks. During the initial 6 weeks of DB treatment, mean change in PANSS total score at endpoint was greater for lurasidone vs. placebo in both the TN group ( -25.0 vs. $-14.4 ; \mathrm{P}<0.02$; effect size, 0.75 ), and in the TP group ( -17.3 vs. $-10.0 ; \mathrm{P}<0.001$; effect size, 0.45 ). During OL extension phase treatment with lurasidone, mean change from DB baseline in the PANSS total score for TN and TP patients, at week 52 was $-32.6(n=38)$ and $-28.1 \quad(n=151)$, respectively; and at week 104 was $-33.6(n=30)$ and -29.2 $(n=126)$, respectively. Mean change from DB baseline in CGI-S score at both weeks 52 and 104 was -1.8 for TN patients and -1.5 for TP patients. At DB baseline mean CGAS scores indicated significant functional impairment in both the TN and TP patients

(CGAS=48 and 43, respectively). During OL treatment with lurasidone, mean change (from DB baseline) in the CGAS score at Weeks 52 and 104, respectively, was +22.0 and +22.9 in TN patients, and +21.1 and +22.9 in TP patients. During OL treatment with lurasidone, mean observed change from DB baseline in the weight (in kg,) at Weeks 52 and 104, respectively, was +4.2 and +4.8 in TN patients, and +4.0 and +5.0 in TP patients. These weight increases are consistent with expected weight gains in adolescents during a 2-year period (based on CDC growth charts).

Conclusions. In this post-hoc analysis of a 2-year study, adolescents with schizophrenia who had received no previous antipsychotic therapy showed greater improvement compared to previously treated patients during both short- and long-term treatment with lurasidone.

Funding. Sunovion Pharmaceuticals Inc.

\title{
Effect of Lurasidone on Manic Symptoms and Treatment- Emergent Mania in Adult and Pediatric Populations with Bipolar Depression
}

Michael Tocco, PhD, Andrei Pikalov, MD, PhD, Courtney Zeni, PhD and Robert Goldman, PhD

Sunovion Pharmaceuticals Inc., Fort Lee, NJ and Marlborough, MA, USA

Presenting Author: Michael Tocco 